\title{
RADIOPROTECTIVE ACTIVITY AND SYNTHESIS OF SILADITHIOACETALS AND GERMADITHIOACETALS DERIVED FROM $N$-SUBSTITUTED NAPHTHYLETHYLIMIDAZOLINE
}

\author{
Benoît Célariès ${ }^{1}$, Ghassoub Rima*1, Louis Court ${ }^{2}$, Claude Lion $^{3}$ \\ and Jean-Denis Laval ${ }^{4}$
}

'Laboratoire d'Hétérochimie Fondamentale et Appliquée, UMR 5069 du CNRS, Université Paul Sabatier, 118, route de Narbonne, F-31062 Toulouse cedex 4, France. Fax : + (33) 561558204

<rima@chimie.ups-tlse.fr>

${ }^{2}$ Service de Radioprotection de l'EDF, 22-28, rue Joubert, F-75009 Paris, France

${ }^{3}$ I.T.O.D.Y.S. Université de Paris VII, Associé au CNRS, 1, rue Guy de la Brosse, F-75005 Paris, France

${ }^{4}$ Unité de Radioprotection, Centre de Recherche du Service de Santé des Armées, 24, avenue des Maquis du Grésivaudan, F-38702 La Tronche cedex, France

\begin{abstract}
A number of organosilicon and organogermanium derivatives and some related compounds including the $N$-substituted 2-[1-naphthylmethyl]-2-imidazoline and 2-[1-(1-naphthyl)ethyl]-2-imidazoline have been prepared and the toxicity of some compounds have been determined in mice. In this paper we report the synthesis and the evaluation of the pharmacological activity of new organosilicon and organogermanium compounds such as sila- and germadithioacetals derived from $N$-substituted naphthylimidazoline.
\end{abstract}

\section{INTRODUCTION}

Recently, it has been reported that medetomidine [1] is a new imidazole drug that possesses selective and potent $\alpha_{2}$-adrenergic properties. $\alpha_{2}$-Adrenergic stimulation is known to mediate a variety of biological actions including hypertension, sedation, antianxiety, analgesia, hypothermia, decreased salivary secretions and mydriasis [2].

The selection of the naphthalene ring system was due to its presence in the potent $\alpha$-adrenergic stimulant naphazoline. The presence of a methyl group attached at the benzylic position of medetomidine is important for optimal $\alpha_{2}$-adrenergic activity [3].

Our group has already reported a considerable work in the field of the chemical radioprotection and pharmacological activity of organosilicon and organogermanium compounds [4-16]. Several organometallic classes derived from $N$-substituted naphthylmethylimidazoline showed a potent radioprotective activity.

Concerning the mechanism of the radioprotective activity of these compounds, it is well established that the main mechanisms of radioprotection are tissual oxygen tension decrease, free radical scavenging and hydrogen transfer [17]. However, the radioprotective action mechanism of naphthylmethylimidazoline remains unclear. Some recent and preliminary investigations seem to indicate that at least in vitro, it has no effect on lipidic peroxidation. Perhaps it acts through its vasoconstrictive effects, which can lower the tissual oxygen supply and hence decrease the radiation-induced lesions.

Another possible explanation of excellent activity of naphthylmethylimidazoline, the first nonsulfur radioprotective compound, is its evolution in vivo of the compound that forms anti-inflammatory products of naphthyl-1-acetic acid type [18]. Further studies are necessary to determine the precise mechanism of action.

Accordingly our objective, we have investigated a new series of organosilicon and organogermanium compounds derived from $N$-substituted 2-[1-(1-naphthyl)ethyl]-2-imidazoline.

\section{Experimental section \\ General procedures}

All manipulations were performed under an inert atmosphere of argon using standard Schlenck, glove box and high-vacuum-line techniques. All solvents used were freshly dried using standard techniques and all glassware was oven-dried. H-NMR spectra were recorded on a Bruker AC 80 spectrometer operating at $80.13 \mathrm{MHz}$ (chemical shifts are reported in parts per million relative to internal $\mathrm{Me}_{4} \mathrm{Si}$ as reference) and ${ }^{13} \mathrm{C}-\mathrm{NMR}$ spectra on an AC 200 spectrometer $(50.32 \mathrm{MHz})$. The multiplicity of the ${ }^{13} \mathrm{C}-\mathrm{NMR}$ signals was determined by the APT technique. Mass spectra under electron impact $(\mathrm{EI})$ or chemical ionisation $\left(\mathrm{CI} / \mathrm{CH}_{4}\right)$ conditions at 70 and $30 \mathrm{eV}$ were obtained on Hewlett-Packard 5989 and Nermag R 10-10H spectrometers. IR and UV spectra were recorded on Perkin-Elmer 1600 FT-IR and Lambda-17 spectrophotometers. 


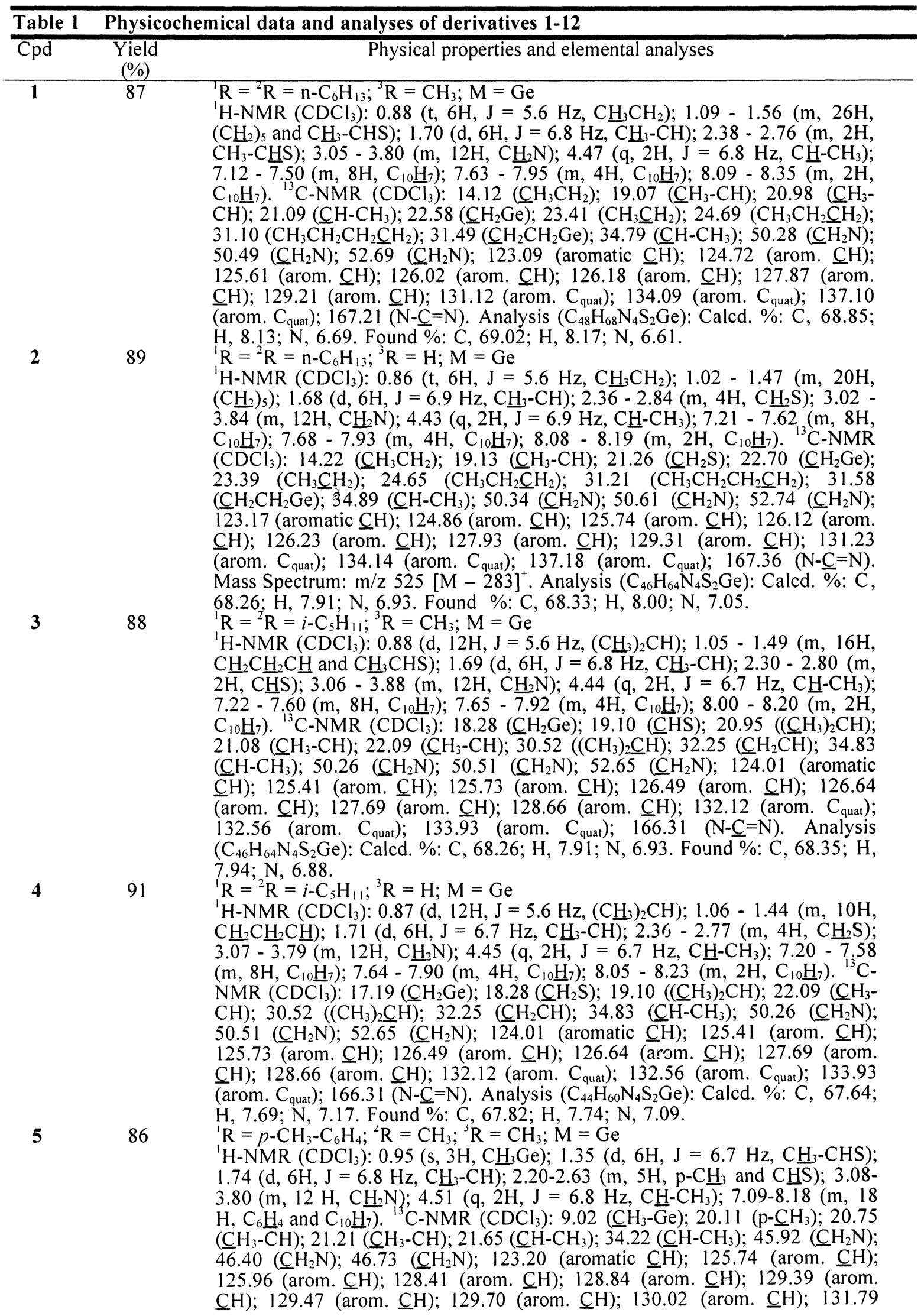


(arom. $\mathrm{C}_{\text {quat }}$ ); 132.26 (arom. $\mathrm{C}_{\text {quat }}$ ); 136.21 (arom. $\mathrm{C}_{\text {quat }}$ ); 140.29 (arom. $\mathrm{C}_{\text {quat }}$ ); 146.49 (arom. $\left.\mathrm{C}_{\text {quat }}\right) ; 170.93(\mathrm{~N}-\mathrm{C}=\mathrm{N})$. Analysis $\left(\mathrm{C}_{44} \mathrm{H}_{52} \mathrm{~N}_{4} \mathrm{~S}_{2} \mathrm{Ge}\right)$ : Calcd. \%: C, 68.16; H, 6.71; N, 7.23. Found \%: C, 68.22; H, 6.87; N, 7.19.

${ }^{1} \mathrm{R}=p-\mathrm{CH}_{3}-\mathrm{C}_{6} \mathrm{H}_{4} ;{ }^{2} \mathrm{R}=\mathrm{CH}_{3} ;{ }^{3} \mathrm{R}=\mathrm{H} ; \mathrm{M}=\mathrm{Ge}$

${ }^{1} \mathrm{H}-\mathrm{NMR}\left(\mathrm{CDCl}_{3}\right): 0.99\left(\mathrm{~s}, 3 \mathrm{H}, \mathrm{CH}_{3} \mathrm{Ge}\right) ; 1.73\left(\mathrm{~d}, 6 \mathrm{H}, \mathrm{J}=6.8 \mathrm{~Hz}, \mathrm{CH}_{3}-\mathrm{CH}\right)$; 2.24-2.58 (m, 7H, p- $\mathrm{CH}_{3}$ and $\left.\mathrm{C}_{2} \mathrm{~S}\right) ; 3.06-3.73\left(\mathrm{~m}, 12 \mathrm{H}, \mathrm{CH}_{2} \mathrm{~N}\right) ; 4.51(\mathrm{q}, 2 \mathrm{H}$, $\left.\mathrm{J}=6.8 \mathrm{~Hz}, \mathrm{CH}-\mathrm{CH}_{3}\right) ; 6.85-8.18\left(\mathrm{~m}, 18 \mathrm{H}, \mathrm{C}_{6} \mathrm{H}_{4}\right.$ and $\left.\mathrm{C}_{10} \underline{\mathrm{H}}_{7}\right) .{ }^{13} \mathrm{C}-\mathrm{NMR}$ $\left(\mathrm{CDCl}_{3}\right)$ : $9.06\left(\mathrm{CH}_{3}-\mathrm{Ge}\right) ; 19.97\left(\mathrm{p}-\mathrm{CH}_{3}\right) ; 21.61\left(\mathrm{CH}_{3}-\mathrm{CH}\right) ; 29.70\left(\mathrm{CH}_{2} \mathrm{~S}\right) ; 34.32$ $\left(\underline{\mathrm{CH}}-\mathrm{CH}_{3}\right) ; 45.84\left(\mathrm{CH}_{2} \mathrm{~N}\right) ; 46.02\left(\mathrm{CH}_{2} \mathrm{~N}\right) ; 47.26\left(\mathrm{CH}_{2} \mathrm{~N}\right) ; 123.20$ (aromatic $\underline{\mathrm{CH}}) ; 125.20$ (arom. $\underline{\mathrm{CH}}) ; 125.78($ arom. $\underline{\mathrm{CH}}) ; 128.62$ (arom. $\underline{\mathrm{CH}}) ; 128.89$ (arom. $\underline{\mathrm{C}}$ ); 129.22 (arom. $\underline{\mathrm{C}} \mathrm{H}) ; 129.36$ (arom. $\underline{\mathrm{CH}}$ ); 129.50 (arom. $\underline{\mathrm{CH}}$ ); 132.23 (arom. $\mathrm{CH}$ ); 133.81 (arom. $\mathrm{C}_{\text {quat }}$ ); 134.07 (arom. $\mathrm{C}_{\text {quat }}$ ); 138.26 (arom. $\left.\mathrm{C}_{\text {quat }}\right) ; 141.35$ (arom. $\mathrm{C}_{\text {quat }}$ ); 147.32 (arom. $\left.\mathrm{C}_{\text {quat }}\right) ; 163.09(\mathrm{~N}-\underline{\mathrm{C}}=\mathrm{N})$. Analysis $\left(\mathrm{C}_{42} \mathrm{H}_{48} \mathrm{~N}_{4} \mathrm{~S}_{2} \mathrm{Ge}\right)$ : Calcd. \%: C, 67.51; H, 6.43; N, 7.50. Found \%: C, 67.44; H, $6.48 ; \mathrm{N}, 7.47$.

${ }^{1} \mathrm{R}={ }^{2} \mathrm{R}=\mathrm{n}-\mathrm{C}_{6} \mathrm{H}_{13} ;{ }^{3} \mathrm{R}=\mathrm{CH}_{3} ; \mathrm{M}=\mathrm{Si}$

'H-NMR $\left(\mathrm{CDCl}_{3}\right): 0.81\left(\mathrm{t}, 6 \mathrm{H}, \mathrm{J}=5.9 \mathrm{~Hz}, \mathrm{CH}_{3} \mathrm{CH}_{2}\right) ; 1.01-1.44(\mathrm{~m}, 26 \mathrm{H}$, $\left(\mathrm{CH}_{2}\right)_{5}$ and $\left.\mathrm{CH}_{3}-\mathrm{CHS}\right) ; 1.75\left(\mathrm{~d}, 6 \mathrm{H}, \mathrm{J}=6.9 \mathrm{~Hz}, \mathrm{C}_{3}-\mathrm{CH}\right) ; 2.36-2.79(\mathrm{~m}, 2 \mathrm{H}$, $\left.\mathrm{CH}_{3}-\mathrm{CHS}\right) ; 3.04-3.81\left(\mathrm{~m}, 12 \mathrm{H}, \mathrm{CH}_{2} \mathrm{~N}\right) ; 4.53\left(\mathrm{q}, 2 \mathrm{H}, \mathrm{J}=6.9 \mathrm{~Hz}, \mathrm{C} \underline{\mathrm{H}}-\mathrm{CH}_{3}\right)$; $7.25-7.56\left(\mathrm{~m}, 8 \mathrm{H}, \mathrm{C}_{10} \mathrm{H}_{7}\right) ; 7.61-7.86\left(\mathrm{~m}, 4 \mathrm{H}, \mathrm{C}_{10} \mathrm{H}_{7}\right) ; 7.90-8.21(\mathrm{~m}, 2 \mathrm{H}$, $\left.\mathrm{C}_{10} \mathrm{H}_{7}\right) \cdot{ }^{13} \mathrm{C}$-NMR $\left(\mathrm{CDCl}_{3}\right): 14.12\left(\mathrm{CH}_{3} \mathrm{CH}_{2}\right) ; 16.18\left(\mathrm{CH}_{2} \mathrm{Si}\right) ; 18.48\left(\mathrm{CH}_{3} \mathrm{CH}_{2}\right)$; $18.67\left(\mathrm{CH}_{3} \mathrm{CH}\right) ; 19.58(\mathrm{CHS}) ; 20.85\left(\mathrm{CH}_{3} \mathrm{CH}\right) ; 22.56\left(\mathrm{CH}_{3} \mathrm{CH}_{2} \mathrm{CH}_{2}\right) ; 25.58$ $\left(\mathrm{CH}_{3} \mathrm{CH}_{2} \mathrm{CH}_{2} \mathrm{CH}_{2}\right) ; 31.58\left(\mathrm{CH}_{2} \mathrm{CH}_{2} \mathrm{Si}\right) ; 33.44\left(\mathrm{C} H-\mathrm{CH}_{3}\right) ; 44.40\left(\mathrm{CH}_{2} \mathrm{~N}\right) ; 46.16$ $\left(\mathrm{CH}_{2} \mathrm{~N}\right) ; 50.13\left(\mathrm{CH}_{2} \mathrm{~N}\right) ; 123.18$ (aromatic $\left.\underline{\mathrm{C}} \mathrm{H}\right) ; 125.38$ (arom. $\left.\underline{\mathrm{C}} \mathrm{H}\right) ; 125.79$ (arom. $\underline{\mathrm{CH}}$ ); 125.99 (arom. $\underline{\mathrm{CH}}$ ); 127.02 (arom. $\underline{\mathrm{C} H}) ; 128.71$ (arom. $\underline{\mathrm{C} H}$ ); 128.87 (arom. $\underline{\mathrm{C}} \mathrm{H}) ; 130.96$ (arom. $\mathrm{C}_{\text {quat }}$ ); 133.68 (arom. $\mathrm{C}_{\text {quat }}$ ); 134.08 (arom. $\left.\mathrm{C}_{\text {quat }}\right) ; 173.80(\mathrm{~N}-\mathrm{C}=\mathrm{N})$. Mass Spectrum: $\mathrm{m} / \mathrm{z} 792[\mathrm{M}]^{+} ; 704[\mathrm{M}-2 \times 44]^{+}$. Analysis $\left(\mathrm{C}_{48} \mathrm{H}_{68} \mathrm{~N}_{4} \mathrm{~S}_{2} \mathrm{Si}\right)$ : Calcd. \%: C, 72.73; H, 8.59; N, 7.07. Found \%: C, $72.69 ; \mathrm{H}, 8.65 ; \mathrm{N}, 7.03$.

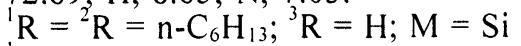

${ }^{1} \mathrm{H}$-NMR $\left(\mathrm{CDCl}_{3}\right): 0.83\left(\mathrm{t}, 6 \mathrm{H}, \mathrm{J}=5.7 \mathrm{~Hz}, \mathrm{CH}_{3} \mathrm{CH}_{2}\right) ; 1.00-1.49(\mathrm{~m}, 20 \mathrm{H}$, $\left.\left(\mathrm{CH}_{2}\right)_{5}\right) ; 1.75\left(\mathrm{~d}, 6 \mathrm{H}, \mathrm{J}=6.3 \mathrm{~Hz}, \mathrm{CH}_{3}-\mathrm{CH}\right) ; 2.36-2.84\left(\mathrm{~m}, 4 \mathrm{H}, \mathrm{CH}_{2} \mathrm{~S}\right) ; 2.95-$ $4.10\left(\mathrm{~m}, 12 \mathrm{H}, \mathrm{CH}_{2} \mathrm{~N}\right) ; 4.56\left(\mathrm{q}, 2 \mathrm{H}, \mathrm{J}=6.3 \mathrm{~Hz}, \mathrm{C} \underline{\mathrm{H}}-\mathrm{CH}_{3}\right) ; 7.28-7.63(\mathrm{~m}, 8 \mathrm{H}$, $\left.\mathrm{C}_{10} \underline{\mathrm{H}}_{7}\right) ; 7.64-7.88\left(\mathrm{~m}, 4 \mathrm{H}, \mathrm{C}_{10} \underline{\mathrm{H}}_{7}\right) ; 7.92-8.26\left(\mathrm{~m}, 2 \mathrm{H}, \mathrm{C}_{10} \underline{\mathrm{H}}_{7}\right) .{ }^{13} \mathrm{C}-\mathrm{NMR}$ $\left(\mathrm{CDCl}_{3}\right): 14.13\left(\mathrm{CH}_{3} \mathrm{CH}_{2}\right) ; 16.21\left(\underline{\mathrm{C}} \mathrm{CH}_{2} \mathrm{Si}\right) ; 18.51\left(\mathrm{CH}_{3} \underline{\mathrm{C}} \mathrm{H}_{2}\right) ; 19.32\left(\underline{\mathrm{CH}}_{2} \mathrm{~S}\right)$; $22.59\left(\mathrm{CH}_{3}-\mathrm{CH}\right) ; 29.70\left(\mathrm{CH}_{3} \mathrm{CH}_{2} \mathrm{CH}_{2}\right) ; 31.61 \quad\left(\mathrm{CH}_{3} \mathrm{CH}_{2} \mathrm{CH}_{2} \mathrm{CH}_{2}\right) ; 32.66$ $\left(\mathrm{CH}_{2} \mathrm{CH}_{2} \mathrm{Si}\right) ; 34.22\left(\underline{\mathrm{C}} \mathrm{H}-\mathrm{CH}_{3}\right) ; 46.02\left(\underline{\mathrm{CH}}_{2} \mathrm{~N}\right) ; 49.88\left(\underline{\mathrm{CH}}_{2} \mathrm{~N}\right) ; 49.99\left(\underline{\mathrm{C}} \mathrm{H}_{2} \mathrm{~N}\right) ;$ 123.28 (aromatic $\mathrm{CH}$ ); 124.75 (arom. $\underline{\mathrm{CH}}$ ); 125.39 (arom. $\underline{\mathrm{CH}}$ ); 125.81 (arom. $\underline{\mathrm{CH}}) ; 126.50$ (arom. $\underline{\mathrm{CH}}$ ); 127.03 (arom. $\mathrm{CH}) ; 128.87$ (arom. $\underline{\mathrm{CH}}$ ); 130.05 (arom. $\mathrm{C}_{\text {quat }}$ ); 131.03 (arom. $\left.\mathrm{C}_{\text {quat }}\right) ; 133.77$ (arom. $\left.\mathrm{C}_{\text {quat }}\right) ; 176.76(\mathrm{~N}-\mathrm{C}=\mathrm{N})$. Analysis $\left(\mathrm{C}_{46} \mathrm{H}_{64} \mathrm{~N}_{4} \mathrm{~S}_{2} \mathrm{Si}\right)$ : Calcd. \%: $\mathrm{C}, 72.25 ; \mathrm{H}, 8.38 ; \mathrm{N}, 7.33$. Found \%: $\mathrm{C}$, $72.29 ; \mathrm{H}, 8.50 ; \mathrm{N}, 7.14$.

${ }^{1} \mathrm{R}={ }^{2} \mathrm{R}=i-\mathrm{C}_{5} \mathrm{H}_{11} ;{ }^{3} \mathrm{R}=\mathrm{CH}_{3} ; \mathrm{M}=\mathrm{Si}$

${ }^{\prime} \mathrm{H}-\mathrm{NMR}\left(\mathrm{CDCl}_{3}\right): 0.86\left(\mathrm{~d}, 12 \mathrm{H}, \mathrm{J}=5.6 \mathrm{~Hz},\left(\mathrm{CH}_{3}\right)_{2} \mathrm{CH}\right) ; 1.04-1.47(\mathrm{~m}, 16 \mathrm{H}$, $\mathrm{CH}_{2} \mathrm{CH}_{2} \mathrm{CH}$ and $\left.\mathrm{CH}_{3} \mathrm{CHS}\right) ; 1.71\left(\mathrm{~d}, 6 \mathrm{H}, \mathrm{J}=6.6 \mathrm{~Hz}, \mathrm{CH}_{3}-\mathrm{CH}\right) ; 2.31-2.97(\mathrm{~m}$, $2 \mathrm{H}, \mathrm{CHS}) ; 3.13-3.84\left(\mathrm{~m}, 12 \mathrm{H}, \mathrm{CH}_{2} \mathrm{~N}\right) ; 4.60\left(\mathrm{q}, 2 \mathrm{H}, \mathrm{J}=6.7 \mathrm{~Hz}, \mathrm{CH}_{-}-\mathrm{CH}_{3}\right) ;$ $7.21-7.61\left(\mathrm{~m}, 8 \mathrm{H}, \mathrm{C}_{10} \mathrm{H}_{7}\right) ; 7.69-7.96\left(\mathrm{~m}, 4 \mathrm{H}, \mathrm{C}_{10} \mathrm{H}_{7}\right) ; 8.01-8.10(\mathrm{~m}, 2 \mathrm{H}$, $\left.\mathrm{C}_{10} \underline{\mathrm{H}}_{7}\right) .{ }^{13} \mathrm{C}$-NMR $\left(\mathrm{CDCl}_{3}\right): 18.19\left(\mathrm{CH}_{2} \mathrm{Si}\right) ; 18.92(\underline{\mathrm{CHS}}) ; 21.15\left(\left(\mathrm{CH}_{3}\right)_{2} \mathrm{CH}\right) ;$ $21.26\left(\mathrm{CH}_{3}-\mathrm{CH}\right) ; 22.15\left(\mathrm{CH}_{3}-\mathrm{CH}\right) ; 30.85\left(\left(\mathrm{CH}_{3}\right)_{2} \mathrm{CH}\right) ; 32.14\left(\mathrm{CH}_{2} \mathrm{CH}\right) ; 34.27$ $\left(\mathrm{CH}-\mathrm{CH}_{3}\right) ; 50.69\left(\mathrm{CH}_{2} \mathrm{~N}\right) ; 51.82\left(\mathrm{CH}_{2} \mathrm{~N}\right) ; 53.44\left(\mathrm{CH}_{2} \mathrm{~N}\right) ; 123.04$ (aromatic $\underline{\mathrm{CH}}) ; 124.89$ (arom. $\underline{\mathrm{CH}}) ; 125.70$ (arom. $\underline{\mathrm{CH}}) ; 126.03$ (arom. $\mathrm{CH}) ; 126.56$ (arom. $\mathrm{CH}) ; 127.99$ (arom. $\mathrm{CH}) ; 128.39$ (arom. $\mathrm{CH}$ ); 131.20 (arom. $\mathrm{C}_{\text {quat }}$ ); 133.91 (arom. $\left.\mathrm{C}_{\text {quat }}\right) ; 135.83$ (arom. $\mathrm{C}_{\text {quat }}$ ); $172.32 \quad(\mathrm{~N}-\mathrm{C}=\mathrm{N})$. Analysis $\left(\mathrm{C}_{46} \mathrm{H}_{64} \mathrm{~N}_{4} \mathrm{~S}_{2} \mathrm{Si}\right)$ : Calcd. \%: C, 72.25; H, 8.38; $\mathrm{N}, 7.33$. Found \%: $\mathrm{C}, 72.17 ; \mathrm{H}$, $8.45 ; \mathrm{N}, 7.29$.

${ }^{1} \mathrm{R}={ }^{\mathrm{N}} \mathrm{R}=i-\mathrm{C}_{5} \mathrm{H}_{11} ;{ }^{3} \mathrm{R}=\mathrm{H} ; \mathrm{M}=\mathrm{Si}$

'H-NMR $\left(\mathrm{CDCl}_{3}\right): 0.86\left(\mathrm{~d}, 12 \mathrm{H}, \mathrm{J}=5.6 \mathrm{~Hz},\left(\mathrm{CH}_{3}\right)_{2} \mathrm{CH}\right) ; 1.07-1.46(\mathrm{~m}, 10 \mathrm{H}$, $\left.\mathrm{CH}_{2} \mathrm{CH}_{2} \mathrm{CH}\right) ; 1.68\left(\mathrm{~d}, 6 \mathrm{H}, \mathrm{J}=6.9 \mathrm{~Hz}, \mathrm{CH}_{3}-\mathrm{CH}\right) ; 2.37-2.79\left(\mathrm{~m}, 4 \mathrm{H}, \mathrm{C}_{2} \mathrm{~S}\right)$; $3.13-3.81\left(\mathrm{~m}, 12 \mathrm{H}, \mathrm{CH}_{2} \mathrm{~N}\right) ; 4.63\left(\mathrm{q}, 2 \mathrm{H}, \mathrm{J}=6.7 \mathrm{~Hz}, \underline{\mathrm{C}}-\mathrm{CH}_{3}\right) ; 7.23-7.61$ $\left(\mathrm{m}, 8 \mathrm{H}, \mathrm{C}_{10} \underline{\mathrm{H}}_{7}\right) ; 7.66-7.90\left(\mathrm{~m}, 4 \mathrm{H}, \mathrm{C}_{10} \underline{\mathrm{H}}_{7}\right) ; 8.00-8.11\left(\mathrm{~m}, 2 \mathrm{H}, \mathrm{C}_{10} \underline{\mathrm{H}}_{7}\right) .{ }^{13} \mathrm{C}-$ NMR $\left(\mathrm{CDCl}_{3}\right): 17.36\left(\mathrm{CH}_{2} \mathrm{Si}\right) ; 18.94\left(\mathrm{C}_{2} \mathrm{~S}\right) ; 20.31\left(\left(\mathrm{CH}_{3}\right)_{2} \mathrm{CH}\right) ; 22.17\left(\mathrm{CH}_{3}-\right.$ $\mathrm{CH}) ; 30.86\left(\left(\mathrm{CH}_{3}\right)_{2} \mathrm{CH}\right) ; 32.37\left(\mathrm{CH}_{2} \mathrm{CH}\right) ; 34.36\left(\underline{\mathrm{C}} \mathrm{H}-\mathrm{CH}_{3}\right) ; 50.03\left(\underline{\mathrm{CH}}_{2} \mathrm{~N}\right)$; $50.24\left(\underline{\mathrm{C}}_{2} \mathrm{~N}\right) ; 51.11\left(\mathrm{CH}_{2} \mathrm{~N}\right) ; 123.81$ (aromatic $\left.\underline{\mathrm{CH}}\right) ; 124.95$ (arom. $\left.\underline{\mathrm{C}} \mathrm{H}\right)$; 
125.56 (arom. $\underline{\mathrm{C}}$ ); 126.34 (arom. $\underline{\mathrm{C} H}$ ); 126.81 (arom. $\underline{\mathrm{CH}}$ ); 127.74 (arom. $\underline{\mathrm{CH}}) ; 128.89$ (arom. $\underline{\mathrm{CH}}$ ); 131.29 (arom. $\mathrm{C}_{\text {quat }}$ ); 133.93 (arom. $\mathrm{C}_{\text {quat }}$ ); 136.53 (arom. $\left.\mathrm{C}_{\text {quat }}\right) ; 171.81(\mathrm{~N}-\mathrm{C}=\mathrm{N})$. Analysis $\left(\mathrm{C}_{44} \mathrm{H}_{60} \mathrm{~N}_{4} \mathrm{~S}_{2} \mathrm{Si}\right)$ : Calcd. \%: C, 71.74; $\mathrm{H}, 8.15 ; \mathrm{N}, 7.61$. Found \%: C, 71.81; H, 8.19; N, 7.56.

${ }^{1} \mathrm{R}=p-\mathrm{CH}_{3}-\mathrm{C}_{6} \mathrm{H}_{4} ;{ }^{2} \mathrm{R}=\mathrm{CH}_{3} ;{ }^{3} \mathrm{R}=\mathrm{CH}_{3} ; \mathrm{M}=\mathrm{Si}$

'H-NMR ( $\left.\mathrm{CDCl}_{3}\right): 0.97\left(\mathrm{~s}, 3 \mathrm{H}, \mathrm{CH}_{3}-\mathrm{Si}\right) ; 1.38\left(\mathrm{~d}, 6 \mathrm{H}, \mathrm{J}=6.8 \mathrm{~Hz}, \mathrm{CH}_{3}-\mathrm{CH}-\mathrm{S}\right)$; $1.71\left(\mathrm{~d}, 6 \mathrm{H}, \mathrm{J}=6.9 \mathrm{~Hz}, \mathrm{CH}_{3}-\mathrm{CH}\right) ; 2.23-2.56\left(\mathrm{~m}, 5 \mathrm{H}, \mathrm{p}-\mathrm{CH}_{3}\right.$ and $\left.\mathrm{CH}-\mathrm{S}\right) ; 3.12-$ $3.70\left(\mathrm{~m}, 12 \mathrm{H}, \mathrm{C}_{2} \mathrm{~N}\right) ; 4.58\left(\mathrm{q}, 2 \mathrm{H}, \mathrm{J}=6.9 \mathrm{~Hz}, \mathrm{C} \underline{\mathrm{H}}-\mathrm{CH}_{3}\right) ; 6.84-8.19(\mathrm{~m}, 18$ $\mathrm{H}, \mathrm{C}_{6} \underline{\mathrm{H}}_{4}$ and $\left.\mathrm{C}_{10} \underline{\mathrm{H}}_{7}\right) .{ }^{3} \mathrm{C}-\mathrm{NMR}\left(\mathrm{CDCl}_{3}\right): 9.07\left(\mathrm{C}_{3}-\mathrm{Si}\right) ; 18.84\left(\mathrm{p}-\mathrm{CH}_{3}\right) ; 19.64$ $\left(\mathrm{CH}_{3}-\mathrm{CH}\right) ; 20.19\left(\mathrm{CH}_{3}-\mathrm{CH}\right) ; 21.64\left(\underline{\mathrm{CH}}-\mathrm{CH}_{3}\right) ; 33.99\left(\mathrm{CH}-\mathrm{CH}_{3}\right) ; 44.51\left(\mathrm{CH}_{2} \mathrm{~N}\right)$; $44.98\left(\mathrm{CH}_{2} \mathrm{~N}\right) ; 46.29\left(\mathrm{CH}_{2} \mathrm{~N}\right) ; 124.96$ (aromatic $\left.\underline{\mathrm{C}} \mathrm{H}\right) ; 125.84$ (arom. $\left.\underline{\mathrm{C}} \mathrm{H}\right)$; 126.02 (arom. $\mathrm{CH}$ ); 127.27 (arom. $\mathrm{CH}$ ); 127.69 (arom. $\mathrm{CH}$ ); 128.42 (arom. $\underline{\mathrm{CH}}) ; 129.06$ (arom. $\underline{\mathrm{CH}}) ; 129.54$ (arom. $\mathrm{CH}) ; 133.16$ (arom. $\underline{\mathrm{CH}}$ ); 133.50 (arom. $\mathrm{C}_{\text {quat }}$ ); 134.12 (arom. $\left.\mathrm{C}_{\text {quat }}\right) ; 136.31$ (arom. $\mathrm{C}_{\text {quat }}$ ); 140.13 (arom. $\mathrm{C}_{\text {quat }}$ ); 143.39 (arom. $\left.\mathrm{C}_{\text {quat }}\right) ; 171.85(\mathrm{~N}-\mathrm{C}=\mathrm{N})$. Analysis $\left(\mathrm{C}_{44} \mathrm{H}_{52} \mathrm{~N}_{4} \mathrm{~S}_{2} \mathrm{Si}\right)$ : Calcd. \%: $\mathrm{C}$, 72.53; H, 7.14; N, 7.69. Found \%: C, 72.47; H, 7.21; N, 7.76.

$1290 \quad{ }^{1} \mathrm{R}=p-\mathrm{CH}_{3}-\mathrm{C}_{6} \mathrm{H}_{4} ;{ }^{2} \mathrm{R}=\mathrm{CH}_{3} ;{ }^{3} \mathrm{R}=\mathrm{H} ; \mathrm{M}=\mathrm{Si}$

${ }^{\prime} \mathrm{H}-\mathrm{NMR}\left(\mathrm{CDCl}_{3}\right): 1.03\left(\mathrm{~s}, 3 \mathrm{H}, \mathrm{CH}_{3}-\mathrm{Si}\right) ; 1.72\left(\mathrm{~d}, 6 \mathrm{H}, \mathrm{J}=7.1 \mathrm{~Hz}, \mathrm{CH}_{3}-\mathrm{CH}\right)$; 2.24-2.60 (m, $7 \mathrm{H}, \mathrm{p}-\mathrm{CH}_{3}$ and $\left.\mathrm{CH}_{2} \mathrm{~S}\right) ; 3.08-3.66\left(\mathrm{~m}, 12 \mathrm{H}, \mathrm{CH}_{2} \mathrm{~N}\right) ; 4.51(\mathrm{q}, 2 \mathrm{H}$, $\left.\mathrm{J}=7.1 \mathrm{~Hz}, \mathrm{CH}-\mathrm{CH}_{3}\right) ; 6.85-8.11\left(\mathrm{~m}, 18 \mathrm{H}, \mathrm{C}_{6} \mathrm{H}_{4}\right.$ and $\left.\mathrm{C}_{10} \underline{\mathrm{H}}_{7}\right) .{ }^{13} \mathrm{C}-\mathrm{NMR}$ $\left(\mathrm{CDCl}_{3}\right): 8.88\left(\mathrm{CH}_{3}-\mathrm{Si}\right) ; 18.79\left(\mathrm{p}-\mathrm{CH}_{3}\right) ; 19.54\left(\mathrm{C}_{3}-\mathrm{CH}\right) ; 22.61\left(\underline{\mathrm{CH}}_{2} \mathrm{~S}\right) ; 34.09$ $\left(\mathrm{CH}-\mathrm{CH}_{3}\right) ; 44.96\left(\mathrm{CH}_{2} \mathrm{~N}\right) ; 45.09\left(\mathrm{CH}_{2} \mathrm{~N}\right) ; 46.13\left(\mathrm{CH}_{2} \mathrm{~N}\right) ; 124.18$ (aromatic $\underline{\mathrm{CH}}$ ); 125.69 (arom. $\underline{\mathrm{CH}}$ ); 126.19 (arom. $\underline{\mathrm{CH}}$ ); 128.41 (arom. $\underline{\mathrm{CH}}$ ); 129.12 (arom. $\mathrm{CH}$ ); 129.81 (arom. $\underline{\mathrm{CH}}$ ); 130.79 (arom. $\underline{\mathrm{CH}}$ ); 131.46 (arom. $\underline{\mathrm{CH}}$ ); 133.18 (arom. $\mathrm{CH}$ ); 134.18 (arom. $\mathrm{C}_{\text {quat }}$ ); 134.41 (arom. $\mathrm{C}_{\text {quat }}$ ); 136.74 (arom. $\left.\mathrm{C}_{\text {quat }}\right) ; 141.08$ (arom. $\left.\mathrm{C}_{\text {quat }}\right) ; 144.38$ (arom. $\left.\mathrm{C}_{\text {quat }}\right) ; 172.01(\mathrm{~N}-\mathrm{C}=\mathrm{N})$. Analysis $\left(\mathrm{C}_{42} \mathrm{H}_{48} \mathrm{~N}_{4} \mathrm{~S}_{2} \mathrm{Si}\right)$ : Calcd. \%: C, 72.00; H, 6.86; N, 8.00. Found \%: C, 72.11; H, $6.83 ; \mathrm{N}, 8.06$.

Melting points were taken uncorrected on a Leitz Biomed hot-plate microscope apparatus or, in capillary tubes, on a digital Electrothermal apparatus. Elemental analyses $(\mathrm{C}, \mathrm{H}, \mathrm{N})$ were performed at the "Laboratoire de Microanalyse de l'Ecole Nationale Supérieure de Chimie" of Toulouse.

Synthesis of metalladithioacetals 1-12

All these compounds were prepared by a method already described.

Siladithioacetal 8 (method A)

To a stirred mixture of freshly distilled triethylamine $(0.96 \mathrm{~g}, 9.5 \mathrm{mmol})$ and 1-(1-thioethyl)-2-[1(1-naphthyl)ethyl]-2-imidazoline $(2.70 \mathrm{~g}, 9.5 \mathrm{mmol})$ in $30 \mathrm{ml}$ of THF was added dropwise with stirring a solution of diisoamyldichlorosilane $(1.28 \mathrm{~g}, 4.75 \mathrm{mmol})$ in $30 \mathrm{ml}$ of THF. The reaction mixture was refluxed under an argon atmosphere for 5 hours. After filtration, the residue was concentrated under vacuum to give $8(3.2 \mathrm{~g}, 88 \%)$.

Germadithioacetal 4 (method B)

A solution of bis(diethylamino)diisoamylgermane $(1.71 \mathrm{~g}, 4.75 \mathrm{mmol})$ in $30 \mathrm{ml}$ of THF was added slowly with stirring to a solution of 1-(1-thioethyl)-2-[1-(1-naphthyl)ethyl]-2-imidazoline (2.70 g, $9.5 \mathrm{mmol})$ in $30 \mathrm{ml}$ of THF. The reaction mixture was refluxed under argon atmosphere for 5 hours. After cooling to room temperature, the volatile material was removed under vacuum to afford $4(3.38 \mathrm{~g}, 91 \%)$.

The physiochemical data and the analyses are reported in Table 1

\section{Synthesis of selenodiazadihexylgermetane 13}

\section{Method C}

To a solution of 1,3-bis(trimethylsilyl)selenourea $(2.2 \mathrm{~g}, 8.23 \mathrm{mmol})$ in $100 \mathrm{ml}$ of THF was added slowly with stirring a solution of bis(diethylamino)dihexylgermane $(3.18 \mathrm{~g}, 8.23 \mathrm{mmol})$ in $10 \mathrm{ml}$ of THF. The reaction mixture was refluxed for 5 hours. After the mixture was cooled to room temperature the volatiles were removed under vacuum to afford $13(1.88 \mathrm{~g}, 45 \%)$ as a yellow oil.

\section{Method D}

To a solution of 1,3-bis(trimethylsilyl)selenourea $(2.2 \mathrm{~g}, 8.23 \mathrm{mmol})$ and triethylamine $(1.67 \mathrm{~g}$, $16.46 \mathrm{mmol}$ ) freshly distilled in $100 \mathrm{ml}$ of THF was added dropwise with stirring a solution of dichlorodihexylgermane $(2.58 \mathrm{~g}, 8.23 \mathrm{mmol})$ in $10 \mathrm{ml}$ of THF. The reaction mixture was refluxed for 5 hours. After cooling to room temperature, the mixture was filtered and the volatiles were removed under vacuum to give $13(1.59 \mathrm{~g}, 38 \%)$.

13: ${ }^{1} \mathrm{H}-\mathrm{NMR}\left(\mathrm{CDCl}_{3}\right): 0.15\left(\mathrm{~s}, 18 \mathrm{H},\left(\mathrm{CH}_{3}\right)_{3} \mathrm{Si}\right) ; 0.86\left(\mathrm{t}, 6 \mathrm{H}, \mathrm{J}=5.6 \mathrm{~Hz}, \mathrm{CH}_{3} \mathrm{CH}_{2}\right) ; 1.10-1.50(\mathrm{~m}, 20 \mathrm{H}$, $\left.\left(\mathrm{CH}_{2}\right)_{5}\right) .{ }^{13} \mathrm{C}-\mathrm{NMR}\left(\mathrm{CDCl}_{3}\right): 1.06\left(\left(\mathrm{CH}_{3}\right)_{3} \mathrm{Si}\right) ; 14.08\left(\mathrm{C}_{3} \mathrm{CH}_{2}\right) ; 22.57\left(\mathrm{CH}_{3} \mathrm{CH}_{2}\right) ; 25.27\left(\mathrm{CH}_{3} \mathrm{CH}_{2} \mathrm{CH}_{2}\right)$; $25.76\left(\mathrm{CH}_{3} \mathrm{CH}_{2} \mathrm{CH}_{2} \mathrm{CH}_{2}\right) ; 31.39\left(\mathrm{CH}_{2} \mathrm{CH}_{2} \mathrm{Ge}\right) ; 32.13\left(\mathrm{CH}_{2} \mathrm{Ge}\right) ; \mathrm{C}=$ Se not detected.

Mass spectrum: $\mathrm{m} / \mathrm{z} 508[\mathrm{M}]^{+} ; 431[\mathrm{M}-\mathrm{Se}+1]^{+}$. Analysis $\left(\mathrm{C}_{19} \mathrm{H}_{44} \mathrm{~N}_{2} \mathrm{SeSi}_{2} \mathrm{Ge}\right):$ Calcd. \%: C, 44.79; $\mathrm{H}$, $8.64 ; \mathrm{N}, 5.50$. Found \%: C, 44.86; H, 8.69; N, 5.49. 


\section{Synthesis of selenodiazadiisoamylgermetane 14}

Using the same experimental procedure as for the synthesis of 13 , the reaction of dichlorodiisoamylgermane $(3.13 \mathrm{~g}, 10.96 \mathrm{mmol})$ in the presence of triethylamine $(2.22 \mathrm{~g}, 21.92 \mathrm{mmol})$ or bis(diethylamino)diisoamylgermane $(3.93 \mathrm{~g}, 10.96 \mathrm{mmol}$ ) with 1,3-bis(trimethylsilyl)selenourea $(2.93 \mathrm{~g}$, $10.96 \mathrm{mmol}$ ) does not lead to 14 but afford only the selenagermaadamantane $15(2.50 \mathrm{~g}, 87 \%)$ as a yellow solid, and 1,3-bis(trimethylsilyl)carbodiimide.

Synthesis of hexaselenatetrakis(isoamylgerma)adamantane 15

A solution of $\mathrm{LiEt}_{3} \mathrm{BH}(57.20 \mathrm{mmol}$ in $57.2 \mathrm{ml}$ of THF) was added dropwise to elemental selenium $(2.26 \mathrm{~g}, 28.60 \mathrm{mmol})$ via syringe. The mixture was stirred for 1 hour at room temperature. A solution of trichloroisoamylgermane $(4.77 \mathrm{~g}, 19.07 \mathrm{mmol})$ in $25 \mathrm{ml}$ of anhydrous THF was added at $0{ }^{\circ} \mathrm{C}$ for 1 hour. After the addition the reaction mixture was warmed to room temperature and stirred until the red colour of the selenium salt had disappeared (5 days). The solvent was removed in vacuo and the residue was extracted with toluene. After filtration and concentration the residue solid was crystallized from pentane to afford $15(4.5 \mathrm{~g}, 90 \%)$.

15: $\left.{ }^{1} \mathrm{H}-\mathrm{NMR}(\mathrm{CDCl})_{3}\right): 0.90\left(\mathrm{~d}, 24 \mathrm{H}, \mathrm{J}=5.4 \mathrm{~Hz},\left(\mathrm{CH}_{3}\right)_{2} \mathrm{CH}\right) ; 1.20-2.11\left(\mathrm{~m}, 20 \mathrm{H}, \mathrm{CH}_{2} \mathrm{CH}_{2} \mathrm{CH}\right) .{ }^{13} \mathrm{C}-\mathrm{NMR}$ $\left(\mathrm{CDCl}_{3}\right): 21.96\left(\mathrm{CH}_{3}\right) ; 29.70(\underline{\mathrm{CH}}) ; 31.37\left(\mathrm{CH}_{2}\right) ; 32.69\left(\mathrm{CH}_{2}\right)$. Mass spectrum $\left(\mathrm{CI}: \mathrm{CH}_{4}\right): \mathrm{m} / \mathrm{z} 1079[\mathrm{M}+$ $29]^{+}$. Analysis $\left(\mathrm{C}_{20} \mathrm{H}_{44} \mathrm{Se}_{6} \mathrm{Ge}_{4}\right)$ : Calcd. \%: $\mathrm{C}, 22.90 ; \mathrm{H}, 4.20$. Found \%: C, 22.93; H, 4.23 .

This compound has been shown by X-Ray crystallographic methods to have an adamantane-type structure but the quality of crystal does not permit us to do a structure refinement.

Synthesis of 2-[1-(1-naphthyl)ethyl]-2-imidazoline 16

a) $\quad \mathrm{N}$-Boc 2-(1-naphthylmethyl)-2-imidazoline :

To a stirred solution of 2-(1-naphthylmethyl)-2-imidazoline $(19 \mathrm{~g}, 90.35 \mathrm{mmol})$ in $200 \mathrm{ml}$ of THF was added dropwise a solution of ditertbutyldicarbonate $(21 \mathrm{ml}, 91.64 \mathrm{mmol})$ in $20 \mathrm{ml}$ of THF. The mixture was stirred at room temperature for 8 hours. The volatiles were removed under vacuum to afford $\mathrm{N}$ Boc 2-(1-naphthylmethyl)-2-imidazoline (25.52 g, $91 \%)$.

${ }^{1} \mathrm{H}-\mathrm{NMR}\left(\mathrm{CDCl}_{3}\right): 1.35\left(\mathrm{~s}, 9 \mathrm{H},\left(\mathrm{C}_{3}\right)_{3} \mathrm{C}\right) ; 3.76\left(\mathrm{~s}, 4 \mathrm{H}, \mathrm{C}_{2} \mathrm{~N}\right) ; 4.49\left(\mathrm{~s}, 2 \mathrm{H}, \mathrm{CH}_{2}-\mathrm{C}_{10} \mathrm{H}_{7}\right) ; 7.33-7.92(\mathrm{~m}, 7 \mathrm{H}$, $\left.\mathrm{C}_{10} \underline{\mathrm{H}}_{7}\right)$.

b) $N$-Boc 2-[1-(1-naphthyl)ethyl]-2-imidazoline :

To a stirred solution of $N$-Boc 2-(1-naphthylmethyl)-2-imidazoline $(25.52 \mathrm{~g}, 82.22 \mathrm{mmol})$ in 300 $\mathrm{ml}$ of THF, at $-78{ }^{\circ} \mathrm{C}$, was added dropwise a solution of n-butyllithium $1.6 \mathrm{M}$ in hexane $(51.4 \mathrm{ml}, 82.22$ $\mathrm{mmol})$. The mixture was stirred at this temperature for 3 hours. A solution of methyliodide $(5.9 \mathrm{ml}, 94.9$ $\mathrm{mmol}$ ) in $30 \mathrm{ml}$ of THF was added dropwise. The mixture was then stirred at room temperature for a night. The volatiles were removed in vacuo and the residue was extracted with of a mixture ether/pentane $(1 / 1,400$ $\mathrm{ml}$ ). After filtration, the solvent was removed under reduced pressure to afford $N$-Boc 2-[1-(1-naphthyl)ethyl]2-imidazoline $(23.86 \mathrm{~g}, 90 \%)$.

${ }^{1} \mathrm{H}-\mathrm{NMR}\left(\mathrm{CDCl}_{3}\right): 0.99\left(\mathrm{~s}, 9 \mathrm{H},\left(\mathrm{CH}_{3}\right)_{3} \mathrm{C}\right) ; 1.41\left(\mathrm{~d}, 3 \mathrm{H}, \mathrm{J}=7.0 \mathrm{~Hz}, \mathrm{CH}_{3}-\mathrm{CH}\right) ; 3.57\left(\mathrm{~s}, 4 \mathrm{H}, \mathrm{CH}_{2} \mathrm{~N}\right) ; 5.15(\mathrm{q}$, $\left.1 \mathrm{H}, \mathrm{J}=7.0 \mathrm{~Hz}, \mathrm{C} \underline{\mathrm{H}}-\mathrm{CH}_{3}\right) ; 7.20-7.90\left(\mathrm{~m}, 7 \mathrm{H}, \mathrm{C}_{10} \underline{\mathrm{H}}_{7}\right) \cdot{ }^{13} \mathrm{C}-\mathrm{NMR}\left(\mathrm{CDCl}_{3}\right): 19.09\left(\mathrm{CH}_{3}-\mathrm{CH}\right) ; 27.87\left(\left(\mathrm{CH}_{3}\right)_{3}-\right.$ C); $35.80\left(\underline{\mathrm{C}} \mathrm{H}-\mathrm{CH}_{3}\right) ; 47.79\left(\mathrm{CH}_{2}-\mathrm{N}\right) ; 51.40\left(\mathrm{CH}_{2}-\mathrm{N}\right) ; 83.05\left(\left(\mathrm{CH}_{3}\right)_{3}-\underline{\mathrm{C}}\right) ; 123.01$ (aromatic $\left.\mathrm{CH}\right) ; 123.30$ (arom. $\mathrm{CH}) ; 125.64$ (arom. $\underline{\mathrm{C} H}) ; 125.75(\operatorname{arom} . \underline{\mathrm{C}} \mathrm{H}) ; 126.36$ (arom. $\mathrm{C} H) ; 127.62$ (arom. $\mathrm{C} H) ; 128.80$ (arom. $\underline{\mathrm{C}} \mathrm{H}$ ); 131.08 (arom. $\mathrm{C}_{\text {quat }}$ ); 133.89 (arom. $\mathrm{C}_{\text {quat }}$ ); 138.10 (arom. $\mathrm{C}_{\text {quat }}$ ); 150.72 (arom. $\mathrm{C}_{\text {quat }}$ ); 165.42 $(\mathrm{N}-\underline{\mathrm{C}}=\mathrm{N})$. Mass spectrum: $\mathrm{m} / \mathrm{z} 324[\mathrm{M}]^{+}$

c) 2-[1-(1-naphthyl)ethyl]-2-imidazoline :

To a stirred solution of $N$-Boc 2-[1-(1-naphthyl)ethyl]-2-imidazoline $(23.86 \mathrm{~g}, 74.00 \mathrm{mmol})$ in 150 $\mathrm{ml}$ of acetic acid was added dropwise a solution of chlorhydric acid $(10 \mathrm{ml}, 36 \%)$. The mixture was stirred at $40{ }^{\circ} \mathrm{C}$ for 5 hours. The mixture was diluted with $400 \mathrm{ml}$ of water and the aqueous layer was extracted with dichloromethane $(4 \times 150 \mathrm{ml})$. The organic layer was washed with a solution of $\mathrm{NaOH}(25 \%)$. The organic layer was then dried over $\mathrm{Na}_{2} \mathrm{SO}_{4}$. Evaporation of solvent in vacuo gave $16(8.63 \mathrm{~g}, 52 \%)$. mp: $160-161^{\circ} \mathrm{C}$. 16: 'H-NMR $\left(\mathrm{CDCl}_{3}\right): 1.70\left(\mathrm{~d}, 3 \mathrm{H}, \mathrm{J}=7.1 \mathrm{~Hz}, \mathrm{CH}_{3}-\mathrm{CH}\right) ; 3.53\left(\mathrm{~s}, 4 \mathrm{H}, \mathrm{CH}_{2} \mathrm{~N}\right) ; 4.39(\mathrm{q}, 1 \mathrm{H}, \mathrm{J}=7.1 \mathrm{~Hz}$, $\left.\mathrm{C} \underline{\mathrm{H}}-\mathrm{CH}_{3}\right) ; 7.09(\mathrm{~s}, 1 \mathrm{H}, \mathrm{N} \underline{\mathrm{H}}) ; 7.40-7.61\left(\mathrm{~m}, 4 \mathrm{H}, \mathrm{C}_{10} \mathrm{H}_{7}\right) ; 7.60-7.81\left(\mathrm{~m}, 2 \mathrm{H}, \mathrm{C}_{10} \underline{\mathrm{H}}_{7}\right) ; 7.82-8.15(\mathrm{~m}, 1 \mathrm{H}$, $\left.\mathrm{C}_{10} \underline{\mathrm{H}}_{7}\right) .{ }^{3} \mathrm{C}-\mathrm{NMR}\left(\mathrm{CDCl}_{3}\right): 18.95\left(\underline{\mathrm{CH}}_{3}-\mathrm{CH}\right) ; 35.50\left(\underline{\mathrm{C}} \mathrm{H}-\mathrm{CH}_{3}\right) ; 47.76\left(\underline{\mathrm{C}} \mathrm{H}_{2}-\mathrm{N}\right) ; 123.08$ (arom. $\left.\underline{\mathrm{C}} \mathrm{H}\right) ; 124.61$ (arom. $\mathrm{CH}) ; 125.63$ (arom. $\underline{\mathrm{C}} \mathrm{H}) ; 125.96$ (arom. $\underline{\mathrm{C}})$ ); 126.67 (arom. $\underline{\mathrm{C}}$ ); 128.13 (arom. $\underline{\mathrm{C}} \mathrm{H}) ; 129.00$ (arom. $\underline{\mathrm{C}} \mathrm{H} 131.24$ (arom. $\mathrm{C}_{\text {quat }}$ ); 133.87 (arom. $\mathrm{C}_{\text {quat }}$ ); 136.71 (arom. $\mathrm{C}_{\text {quat }}$ ); $171.80(\mathrm{~N}-\mathrm{C}=\mathrm{N}) . \mathrm{C} . \mathrm{R} .\left(\mathrm{cm}^{-1}\right)$ ): $v_{\mathrm{NH}}=3430$. Mass spectrum: $\mathrm{m} / \mathrm{z} 223[\mathrm{M}-1]^{+}$. Analysis $\left(\mathrm{C}_{15} \mathrm{H}_{16} \mathrm{~N}_{2}\right):$ Calcd. \%: C, 80.36; H, 7.14; N, 12.50 . Found \%: C, 80.70; H, 7.21; N, 12.09 .

Synthesis of 1-(1-thioethyl)-2-[1-(1-naphthyl)ethyl|-2-imidazoline 17

A solution of 2-[1-(1-naphthyl)ethyl]-2-imidazoline $16(3.66 \mathrm{~g}, 16.32 \mathrm{mmol})$ in $60 \mathrm{ml}$ of dry toluene was mixed with a solution of ethylene sulfide $(1.15 \mathrm{ml}, 19.58 \mathrm{mmol})$ in $20 \mathrm{ml}$ of dry toluene (sealed tube, argon flushed). The reaction mixture was then heated $\left(110^{\circ} \mathrm{C}\right.$ oven) for 22 hours. After cooling, $100 \mathrm{ml}$ of cold diethyl ether was added with stirring to the reaction mixture and a small amount of polyethylene sulfide was filtered. The solvent was removed under reduced pressure to give a yellow pasty product $17(3.25 \mathrm{~g}, 70 \%)$.

17: ' ${ }^{\mathrm{H}-\mathrm{NMR}}\left(\mathrm{CDCl}_{3}\right): 1.68\left(\mathrm{~d}, 3 \mathrm{H}, \mathrm{J}=6.8 \mathrm{~Hz}, \mathrm{CH}_{3}-\mathrm{CH}\right) ; 2.43(\mathrm{~s}, 1 \mathrm{H}, \mathrm{S} \underline{\mathrm{H}}) ; 2.54-2.84\left(\mathrm{~m}, 2 \mathrm{H}, \mathrm{C}_{2}-\mathrm{S}\right)$; $3.02-3.91\left(\mathrm{~m}, 6 \mathrm{H}, \mathrm{CH}_{2} \mathrm{~N}\right) ; 4.41\left(\mathrm{q}, 1 \mathrm{H}, \mathrm{J}=6.8 \mathrm{~Hz}, \underline{\mathrm{C}} \underline{\mathrm{H}}-\mathrm{CH}_{3}\right) ; 7.38-7.50\left(\mathrm{~m}, 4 \mathrm{H}, \mathrm{C}_{10} \underline{\mathrm{H}}_{7}\right) ; 7.58-7.79(\mathrm{~m}, 2 \mathrm{H}$, 
$\left.\mathrm{C}_{10} \underline{\mathrm{H}}_{7}\right) ; 7.80-8.13\left(\mathrm{~m}, 1 \mathrm{H}, \mathrm{C}_{10} \underline{\mathrm{H}}_{7}\right) .{ }^{13} \mathrm{C}-\mathrm{NMR}\left(\mathrm{CDCl}_{3}\right): 19.09\left(\underline{\mathrm{CH}}_{3}-\mathrm{CH}\right) ; 23.40\left(\underline{\mathrm{CH}}_{2}-\mathrm{S}\right) ; 35.12\left(\underline{\mathrm{C}} \mathrm{H}-\mathrm{CH}_{3}\right) ;$ $48.85\left(\mathrm{CH}_{2}-\mathrm{N}\right) ; 49.09\left(\mathrm{CH}_{2}-\mathrm{N}\right) ; 52.41\left(\underline{\mathrm{CH}}_{2}-\mathrm{N}\right) ; 122.81$ (arom. $\left.\underline{\mathrm{C}} \mathrm{H}\right) ; 124.54$ (arom. $\left.\underline{\mathrm{CH}}\right) ; 125.43$ (arom. $\underline{\mathrm{CH}}$ ); 125.80 (arom. $\mathrm{CH}$ ); 126.09 (arom. $\underline{\mathrm{C}} \mathrm{H}$ ); 127.78 (arom. $\underline{\mathrm{CH}}$ ); 129.12 (arom. $\underline{\mathrm{CH}}$ ); 131.04 (arom. $\mathrm{C}_{\text {quat }}$ ); $133.85\left(\right.$ arom. $\left.\mathrm{C}_{\text {quat }}\right) ; 136.67$ (arom. $\mathrm{C}_{\text {quat }}$ ); $168.36(\mathrm{~N}-\mathrm{C}=\mathrm{N})$. I.R. $\left(\mathrm{cm}^{-1}\right): v_{\mathrm{SH}}=2542$. Mass spectrum: $\mathrm{m} / \mathrm{z}$ $283[\mathrm{M}-1]^{+}$. Analysis $\left(\mathrm{C}_{17} \mathrm{H}_{20} \mathrm{~N}_{2} \mathrm{~S}\right)$ : Calcd. \%: $\mathrm{C}, 71.83 ; \mathrm{H}, 7.04 ; \mathrm{N}, 9.86$. Found \%: $\mathrm{C}, 71.89 ; \mathrm{H}, 7.17 ; \mathrm{N}$, 9.77 .

Synthesis of 1-(2-thiopropyl)-2-[1-(1-naphthyl)ethyl]imidazoline 18

Using the same experimental procedure as for the synthesis of 17 , the reaction of $16(3.66 \mathrm{~g}, 16.32$ mmol) with propylene sulfide $(1.29 \mathrm{ml}, 16.40 \mathrm{mmol})$ leads to a yellow pasty product $18(3.17 \mathrm{~g}, 65 \%)$.

18: ${ }^{1} \mathrm{H}-\mathrm{NMR}\left(\mathrm{CDCl}_{3}\right): 1.24\left(\mathrm{~d}, 3 \mathrm{H}, \mathrm{J}=6.6 \mathrm{~Hz}, \mathrm{CH}_{3}-\mathrm{CH}\right) ; 1.65\left(\mathrm{~d}, 3 \mathrm{H}, \mathrm{J}=6.9 \mathrm{~Hz}, \mathrm{CH}_{3}-\mathrm{CH}\right) ; 2.48(\mathrm{~s}, 1 \mathrm{H}$, $\mathrm{S} \underline{\mathrm{H}}) ; 2.58-2.86\left(\mathrm{~m}, 1 \mathrm{H}, \mathrm{C} \underline{\mathrm{H}}-\mathrm{CH}_{3}\right) ; 3.06-4.02\left(\mathrm{~m}, 6 \mathrm{H}, \mathrm{C} \underline{\mathrm{H}}_{2} \mathrm{~N}\right) ; 4.46\left(\mathrm{q}, 1 \mathrm{H}, \mathrm{J}=6.9 \mathrm{~Hz}, \mathrm{C}^{\mathrm{H}}-\mathrm{CH}_{3}\right) ; 7.11-7.52$ $\left(\mathrm{m}, 4 \mathrm{H}, \mathrm{C}_{10} \mathrm{H}_{7}\right) ; 7.66-7.82\left(\mathrm{~m}, 2 \mathrm{H}, \mathrm{C}_{10} \mathrm{H}_{7}\right) ; 7.84-8.16\left(\mathrm{~m}, 1 \mathrm{H}, \mathrm{C}_{10} \mathrm{H}_{7}\right) .{ }^{13} \mathrm{C}-\mathrm{NMR}\left(\mathrm{CDCl}_{3}\right): 19.13\left(\mathrm{CH}_{3}-\mathrm{CH}\right)$; $21.75\left(\underline{\mathrm{C}} \mathrm{H}-\mathrm{CH}_{3}\right) ; 23.08\left(\underline{\mathrm{CH}}_{3}-\mathrm{CH}\right) ; 35.17\left(\underline{\mathrm{C}} \mathrm{H}-\mathrm{CH}_{3}\right) ; 48.89\left(\mathrm{CH}_{2}-\mathrm{N}\right) ; 49.21\left(\underline{\mathrm{CH}}_{2}-\mathrm{N}\right) ; 52.46\left(\underline{\mathrm{CH}}_{2}-\mathrm{N}\right) ; 123.01$ (arom. $\underline{\mathrm{C}} \mathrm{H}) ; 124.61$ (arom. $\underline{\mathrm{CH}}) ; 125.51($ arom. $\underline{\mathrm{C}} \mathrm{H}) ; 125.90$ (arom. $\underline{\mathrm{C}} \mathrm{H}) ; 126.13$ (arom. $\underline{\mathrm{C}} \mathrm{H}) ; 127.83$ (arom. $\underline{\mathrm{C}} \mathrm{H}$ ); 129.19 (arom. $\underline{\mathrm{CH}}$ ); 131.09 (arom. $\mathrm{C}_{\text {quat }}$ ); 134.06 (arom. $\mathrm{C}_{\text {quat }}$ ); 137.02 (arom. $\mathrm{C}_{\text {quat }}$ ); 168.61 $(\mathrm{N}-\mathrm{C}=\mathrm{N})$. I.R. $\left(\mathrm{cm}^{-1}\right): v_{\mathrm{SH}}=2548$. Mass spectrum: $\mathrm{m} / \mathrm{z} 297[\mathrm{M}-1]^{+}$. Analysis $\left(\mathrm{C}_{18} \mathrm{H}_{22} \mathrm{~N}_{2} \mathrm{~S}\right):$ Calcd. \%: C, $72.48 ; \mathrm{H}, 7.38 ; \mathrm{N}, 9.40$. Found \%: C, 72.57; H, 7.61; N, 9.32.

Synthesis of 1, 3-bis(trimethylsilyl)selenourea 19

To a stirred solution of selenourea $(10 \mathrm{~g}, 81.29 \mathrm{mmol})$ and triethylamine $(36 \mathrm{ml}, 186.96 \mathrm{mmol})$ in $200 \mathrm{ml}$ of THF was added dropwise a solution of chlorotrimethylsilane $(20.6 \mathrm{ml}, 81.29 \mathrm{mmol})$ in $50 \mathrm{ml}$ of THF. The reaction mixture was refluxed under an argon atmosphere for 8 hours. After filtration, the residue was concentrated in vacuo to afford $19(18.47 \mathrm{~g}, 85 \%)$. mp: $146-148{ }^{\circ} \mathrm{C}$ (dec.).

19: ' $\mathrm{H}-\mathrm{NMR}\left(\mathrm{CDCl}_{3}\right): 0.35\left(\mathrm{~s}, 18 \mathrm{H},\left(\mathrm{CH}_{3}\right)_{3} \mathrm{Si}\right) ; 6.20(\mathrm{~s}, 2 \mathrm{H}, \mathrm{NH}) \cdot{ }^{13} \mathrm{C}-\mathrm{NMR}\left(\mathrm{CDCl}_{3}\right): 1.02\left(\left(\mathrm{CH}_{3}\right)_{3} \mathrm{Si}\right)$; $\mathrm{C}=$ Se not detected. Mass spectrum: $\mathrm{m} / \mathrm{z} 267[\mathrm{M}]^{+}$. Analysis $\left(\mathrm{C}_{7} \mathrm{H}_{20} \mathrm{~N}_{2} \mathrm{SeSi}_{2}\right)$ : Calcd. \%: $\mathrm{C}, 31.34 ; \mathrm{H}, 7.46$; N, 10.45. Found \%: C, 31.41; H, 7.51; N, 10.41.

\section{Pharmacology: evaluation of radioprotection}

Three- month-old male mice (Swiss, France), 25 g body weight, were used.

The radioprotective effect of compounds was evaluated by determining the dose reduction factor (DRF), defined as the ratio of $50 \%$ lethal-dose irradiation 30 days $\left(\mathrm{LD}_{50} / 30\right.$ days) of injected mice to that of control mice. Initially the survival rate was determined 30 days after irradiation in different groups of 10 mice receiving an intraperitoneal (i.p.) injection of the test compound with a dose equal to half or one-eighth of its $L_{50} 15$ or 90 min before whole-body irradiation delivered with a dose equal to the $L D_{100} / 30$ days of control mice $(7.5,7.75$ or 8 Gy according to the irradiation date), or with a dose equal to this dose +2 Gy. When necessary, other irradiation doses were tested in order to evaluate the irradiation $\mathrm{LD}_{50}$ of protected mice by the Kraber method (calculated or graphic). [19]

The radiosensitivity of the strain was regularly monitored by the determination of lethality curves of males and females. The $\mathrm{LD}_{50} / 30$ days was between $6.5 \pm 0.3$ and $6.75 \pm 0.3 \mathrm{~Gy}$ according to the date $(\mathrm{P}<$ $0.05)$. Under these conditions significant protection was observed with a DRF value superior to 1.15 .

The toxicity was evaluated by a Probit analysis of the $\mathrm{LD}_{50},[20-21]$ the dose range being determined in a preliminary study. Five groups of ten mice were then injected with different doses within this range.

\section{RESULTS AND DISCUSSION}

Synthesis of metalladithioacetals ${ }^{1}{ }^{2} \mathrm{RM}\left[\mathrm{SCH}\left({ }^{3} \mathrm{R}\right) \mathrm{CH}_{2}-\mathrm{NEI}\right]_{2}$

$\mathrm{NEI}=2$-[1-(1-naphthyl)ethyl]-2-imidazoline

The synthesis of sila- and germadithioacetals compounds derived from $N$-substituted 2-[1-(1naphthyl)ethyl]-2-imidazoline was realised by two methods, $\mathrm{A}$ and $\mathrm{B}[4,22]$.

\section{Method A}

The action of the dichlorodiorganometallane [22] on two equivalents of $N$-substituted naphthylimidazoline in refluxing anhydrous tetrahydrofuran in the presence of freshly distilled triethylamine gave the acyclic derivatives (Scheme 1) in yields of $85-90 \%$.

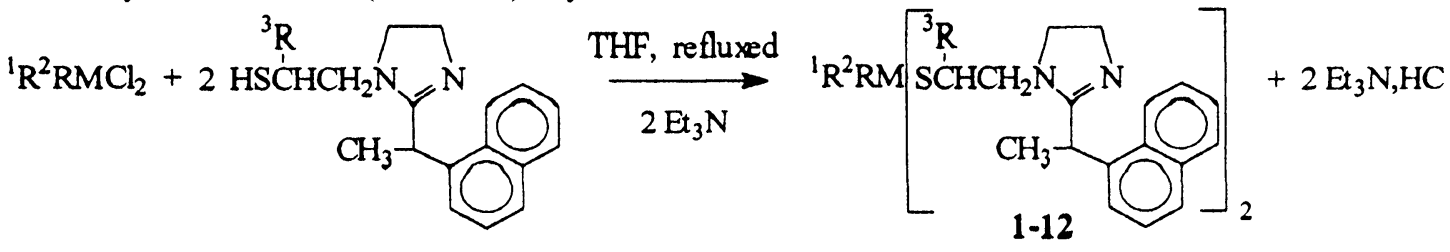

Scheme 1 


\section{Method B}

The reaction of two equivalents of $N$-substituted naphthylimidazoline with the bis(diethylamino)dialkylmetallane in anhydrous tetrahydrofuran (a cleavage of $\mathrm{M}-\mathrm{N}$ bonds by the $\mathrm{SH}$ groups) $[4,22,23]$ gave the corresponding organometallated derivatives (Scheme 2 ) in yield of $92-96 \%$.

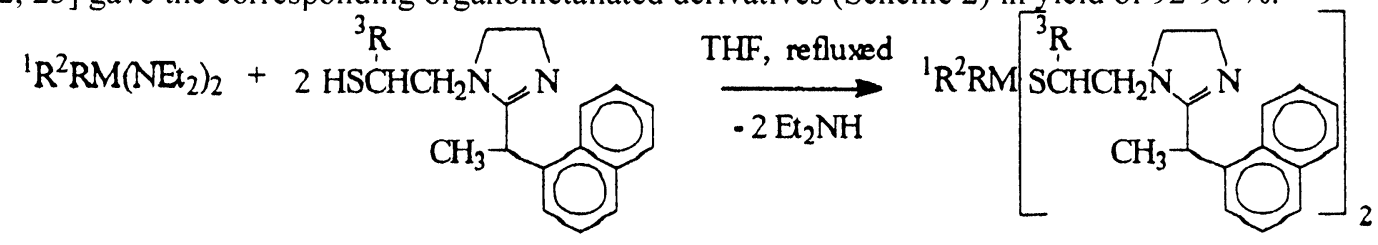

Scheme 2

\section{$1-12$}

$$
\begin{aligned}
& \mathrm{M}=\mathrm{Si} \\
& { }^{1} \mathrm{R}={ }^{2} \mathrm{R}=\mathrm{n}-\mathrm{C}_{6} \mathrm{H}_{13} ;{ }^{3} \mathrm{R}=\mathrm{CH}_{3} \\
& { }^{\mathrm{R}} \mathrm{R}={ }^{2} \mathrm{R}=\mathrm{n}-\mathrm{C}_{6} \mathrm{H}_{13} ;{ }^{3} \mathrm{R}=\mathrm{H} \\
& { }^{\mathrm{I}} \mathrm{R}={ }^{2} \mathrm{R}=i-\mathrm{C}_{5} \mathrm{H}_{11} ;{ }^{3} \mathrm{R}=\mathrm{CH}_{3} \\
& { }^{\mathrm{I}} \mathrm{R}={ }^{2} \mathrm{R}=i-\mathrm{C}_{5} \mathrm{H}_{11} ;{ }^{3} \mathrm{R}=\mathrm{H} \\
& { }^{1} \mathrm{R}=\mathrm{p}-\mathrm{CH}_{3}-\mathrm{C}_{6} \mathrm{H}_{4},{ }^{2} \mathrm{R}=\mathrm{CH}_{3} ;{ }^{3} \mathrm{R}=\mathrm{CH}_{3} \\
& { }^{1} \mathrm{R}=\mathrm{p}-\mathrm{CH}_{3}-\mathrm{C}_{6} \mathrm{H}_{4},{ }^{2} \mathrm{R}=\mathrm{CH}_{3} ;{ }^{3} \mathrm{R}=\mathrm{H}
\end{aligned}
$$

\section{Synthesis of sele nodiazage rme tane $\mathrm{R}_{2} \mathrm{Ge-N}\left(\mathrm{SiMe}_{3}\right)(\mathrm{C}=\mathrm{Se}) \mathrm{N}\left(\mathrm{SiMe}_{3}\right)$}

\section{- $\quad$ Case $\mathrm{R}=\mathrm{n}-\mathrm{C}_{6} \mathrm{H}_{13}$ :}

This compound was also synthesised by two methods, $C$ and $D$.

\section{Method C}

Treatment of 1,3-bis(trimethylsilyl)selenourea, in stoichiometric amounts, with bis(diethylamino)dihexylgermane in refluxing anhydrous tetrahydrofuran resulted with a cleavage of Ge-N bonds by the $\mathrm{NH}$ groups (a transamination reaction) [22-26] forming the corresponding diazagermetane (Scheme 3 ) in yield of $45 \%$.

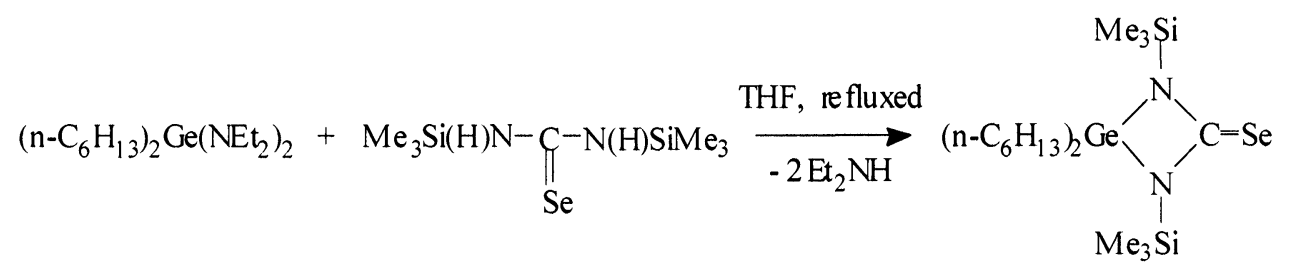

Scheme 3

\section{Method D}

The action of dichlorodihexylgermane, in stoichiometric amounts, on 1,3-bis(trimethylsilyl)selenourea, in refluxing anhydrous tetrahydrofuran in the presence of freshly distilled triethylamine gave by a cyclization reaction, with elimination of hydrochloric acid from $\mathrm{Ge}-\mathrm{Cl}$ and $\mathrm{NH}[22,26]$ group, the corresponding product (Scheme 4 ) in yield of $38 \%$.

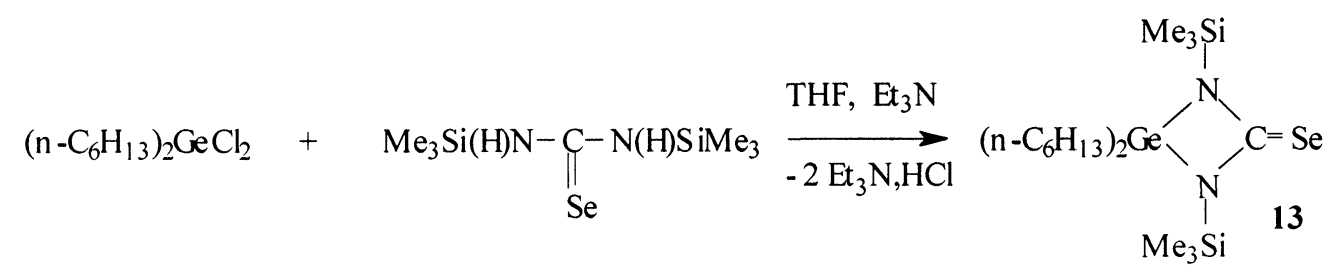

- $\quad$ Case $\mathrm{R}=i-\mathrm{C}_{5} \mathrm{H}_{11}$ :

Scheme 4 
Using the same procedures described above, the reaction between bis(diethylamino)diisoamylgermane or dichlorodiisoamylgermane and 1,3-bis(trimethylsilyl)selenourea does not lead to stable heterocycle but gives only the selenagermaadamantane 15 and 1,3-bis(trimethylsilyl)carbodiimide probably via the intermediate selenodiazagermetane. (Scheme 5).

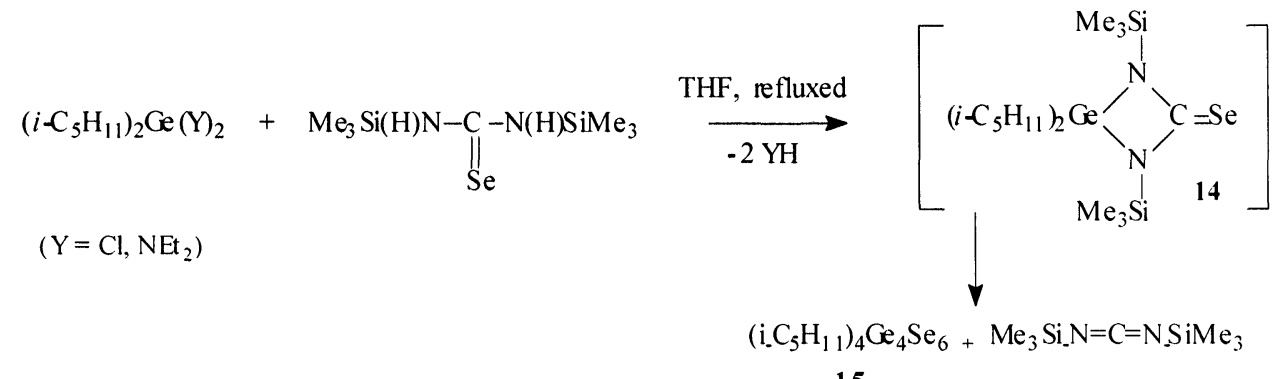

Scheme 5

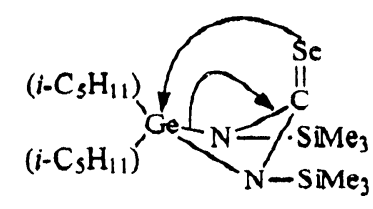

14

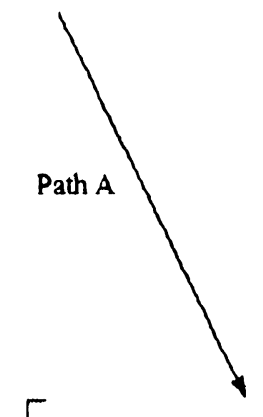

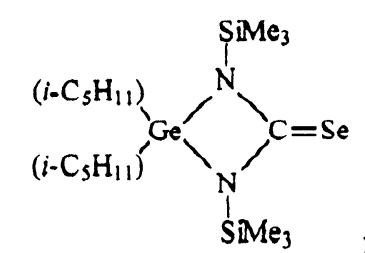<smiles>[Mg][AsH2]</smiles>

14

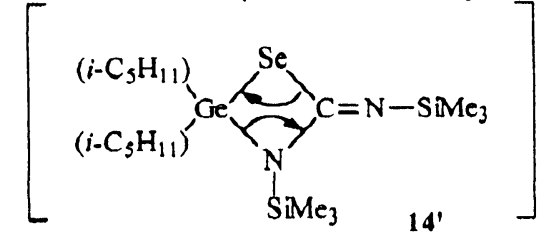

$\downarrow$

$\left[\left(i-\mathrm{C}_{5} \mathrm{H}_{11}\right)_{2} \mathrm{Ge}=\mathrm{Se}\right]+\mathrm{Me}_{3} \mathrm{Si}-\mathrm{N}=\mathrm{C}=\mathrm{N}-\mathrm{SiMe}_{3}$<smiles>[3H]C</smiles>

$\left(i-\mathrm{C}_{5} \mathrm{H}_{11}\right)_{4} \mathrm{Ge}_{4} \mathrm{Se}_{6}$

15

Scheme 6

\section{Reaction mechanism}

The formation of the selenagermaadamantane may be explained by two ways as follows (Scheme 6). 
- Path A: The nucleophilic intramolecular attack of selenium on the germanium atom leads to a corresponding intermediate 14'. Then, this cyclic intermediate 14' undergoes a decomposition to give the germaneselone and 1,3-bis(trimethylsilyl)carbodiimide.

- Path B: The ring cleavage of $\mathbf{1 4}$ results in the formation of the monomeric species [ $i$ $\left.\left.\mathrm{C}_{5} \mathrm{H}_{11}\right)_{2} \mathrm{Ge}=\mathrm{N}-\mathrm{SiMe}_{3}\right]$ and $\left[\mathrm{Me}_{3} \mathrm{Si}-\mathrm{N}=\mathrm{C}=\mathrm{Se}\right]$ as an intermediate, which submits to a pseudo Wittig reaction to afford the intermediate $1 \mathbf{1 4}^{\prime}$.

The germaneselone derivative showed a rearrangement to give preferentially the selenagermaadamantane $\mathbf{1 5}$ which has been completely characterised by usual spectroscopies, mass spectrometry and X-ray diffraction structure.

\section{Molecular modelisation study (ESSF field)}

This study showed the very important steric bulk of the isoamyl groups. The heterocycle $\mathbf{1 3}$ seems to benefit a protection of the reactive centre induced by the hexyl groups.
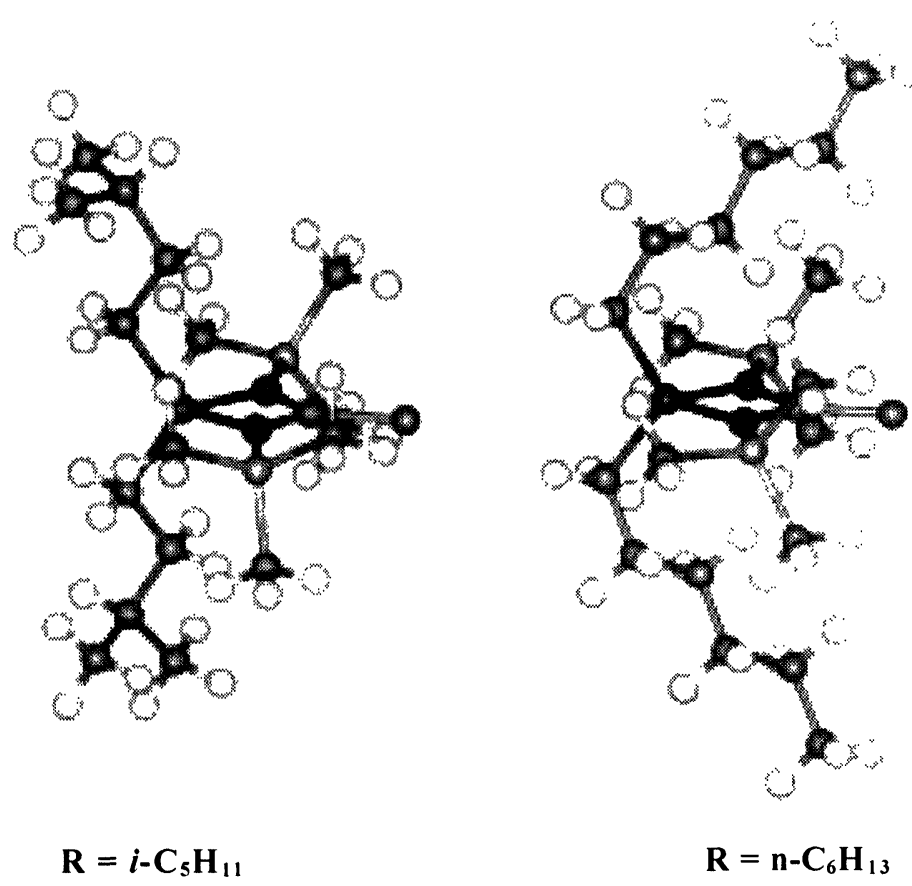

\section{Synthesis of selenagermaadamantane 15}

The treatment of isoamyltrichlorogermane with lithium selenide gave tetragermahexaselenide $\mathbf{1 5}$ [27]. (Scheme 7). The structure was determined by X-ray crystallography.

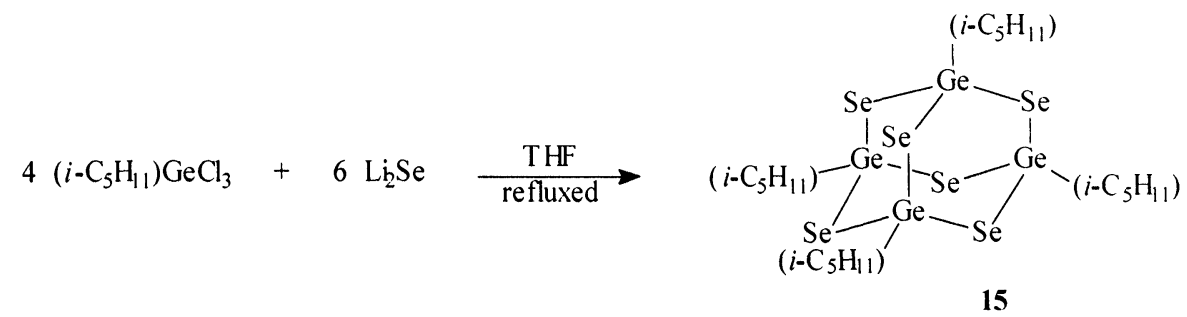

Sc heme 7

Synthesis of the 2-[1-(1-naphthyl)ethyl|-2-imidazoline 16

This product was synthesised according to the following reaction scheme. (Scheme 8). 


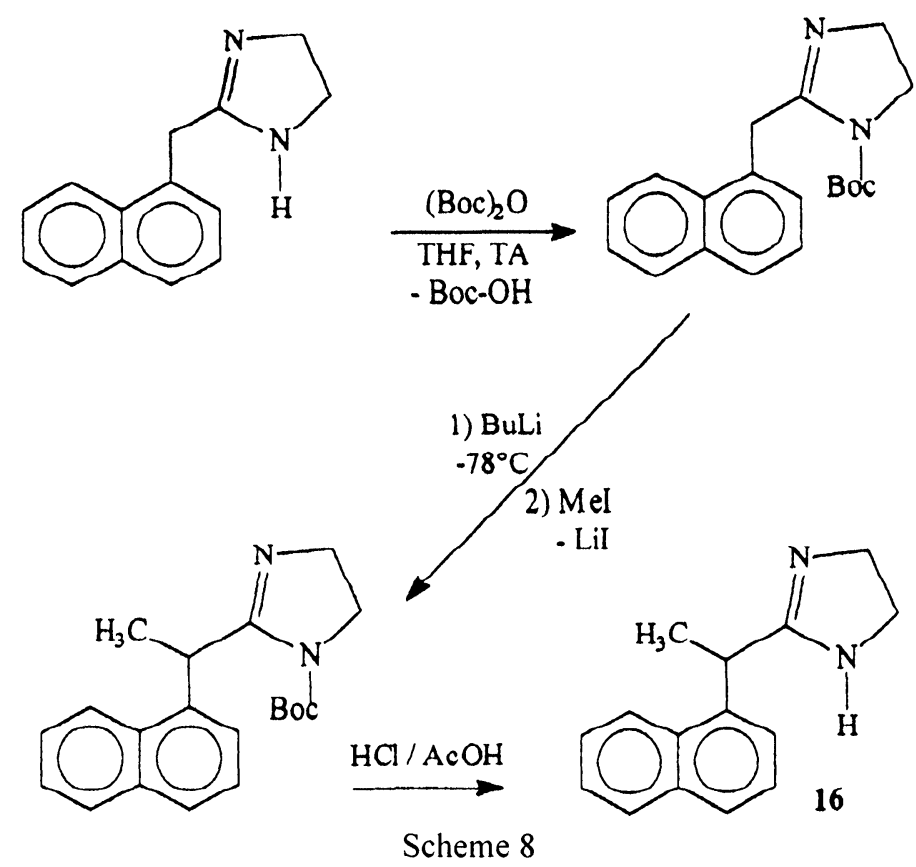

\section{Synthesis of 17 and 18}

The stoichiometric addition of thiirane or methylthiirane to 16 in toluene at $110{ }^{\circ} \mathrm{C}$ leads respectively to 1-(1-thioethyl)-2-[1-(1-naphthyl)ethyl]-2-imidazoline 17 and 1-(2-thiopropyl)-2-[1-(1naphthyl)ethyl]-2-imidazoline 18 (i.e. by a cleavage of the C-S bond by the $\mathrm{NH}$ group [28]) (Scheme 9) in yields of 70 and $65 \%$.<smiles>CN=C(C)C(C)c1cccc2ccccc12</smiles><smiles>[CH2+]N1C=NCC1</smiles><smiles>[R]C1CS1</smiles><smiles>[13CH3][14CH3]</smiles><smiles>[R]C(O)CC1=NCCN1C(C)c1cccc2ccccc12</smiles>

$$
\begin{array}{ll}
\mathrm{R}=\mathrm{H} & 17 \\
\mathrm{R}=\mathrm{Me} & 18
\end{array}
$$

\section{Scheme 9}

\section{Synthesis of 1,3-bis(trimethylsilyl)selenourea 19}

The reaction between equimolar amounts of selenourea and chlorotrimethylsilane in the presence of triethylamine in anhydrous refluxing tetrahydrofuran gives 19 (Scheme 10 ) in yield of $85 \%$.

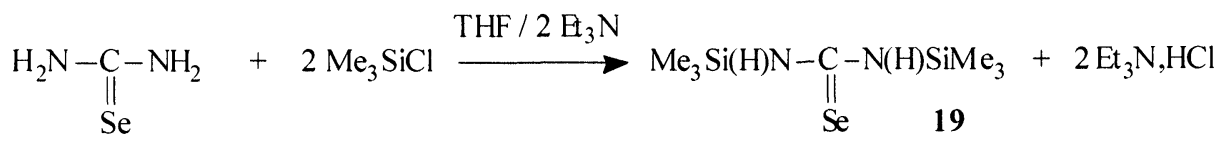

Scheme 10 


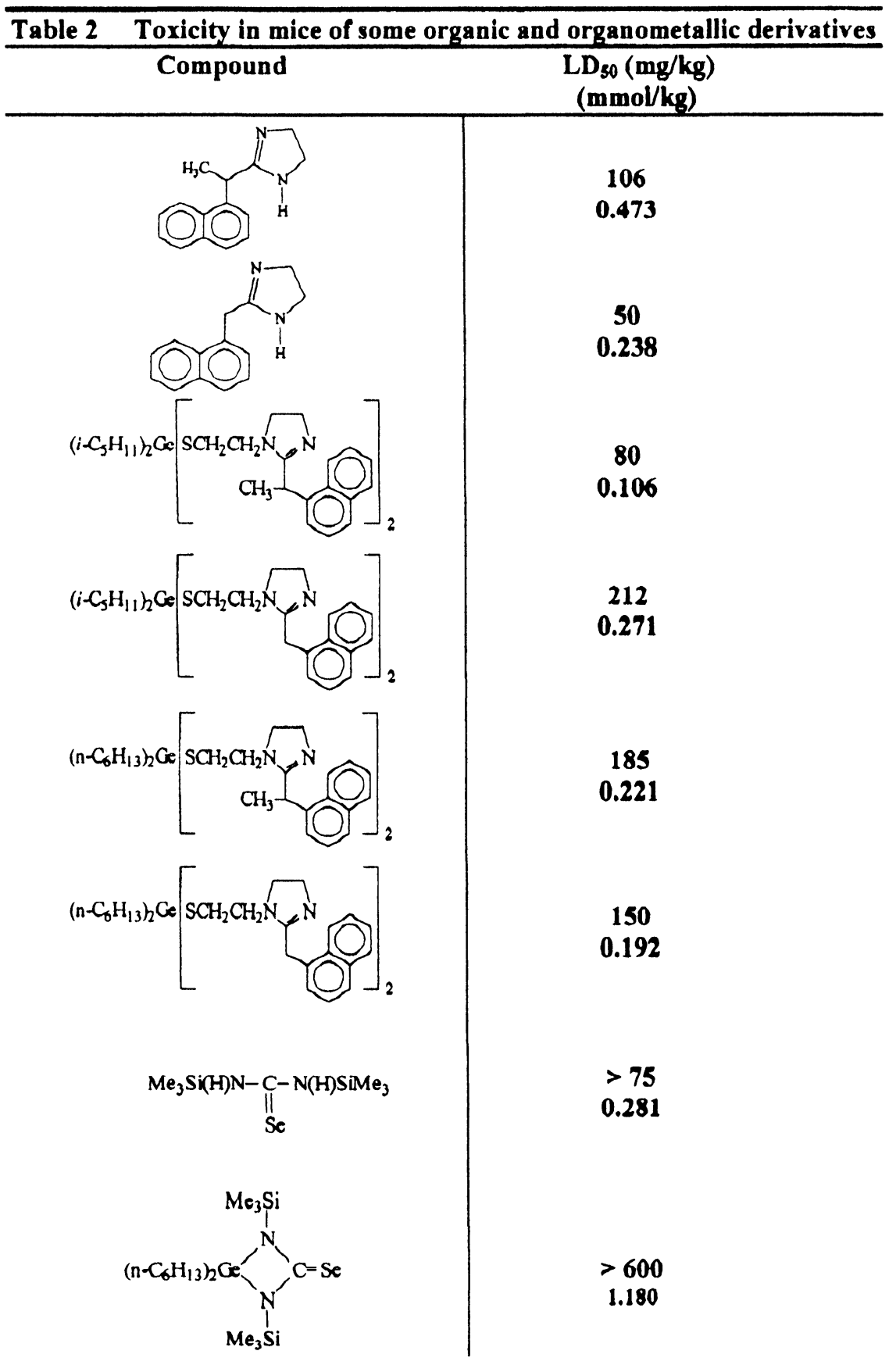

Evaluation of the toxicity

The toxicity obtained with the different compounds has been summarized in table 2 . The biological protocol has been detailed in the Experimental section.

The 2-[1-(1-naphthyl)ethyl]-2-imidazoline (NEI) compounds seems to be less toxic than their 2-(1naphthylmethyl)-2-imidazoline (NMI) homologous. For example, NEI (16) show a two times lower molar toxicity than NMI. The lower toxicity of organometallic compounds compared with basic materials is confirmed by comparison between the toxicity of 13 and 19. Indeed compound 13 is eight times less toxic $\left(\mathrm{LD}_{50}>600 \mathrm{mg} / \mathrm{Kg}\right)$ than the starting derivative $19\left(\mathrm{LD}_{50}>75 \mathrm{mg} / \mathrm{Kg}\right)$. This means that the organogermanium compound 13 have a toxicity four times lower than 19 one (expressed in mol fractions).

The results of the radioprotective activity will be reported at a later date. 


\section{Acknowledgements}

The authors wish to thanks the Délégation Générale pour l'Armement (D.G.A.), Département de Chimie-Pharmacologie, Ministère de la Défense Nationale, France and we would also like to thank the Comité de Radioprotection de l'EDF, France, for their financial support and interest in this research.

\section{References.}

1. H. Scheinin, R. Virtanen, E. Macdonald, R. Lammintausta, M. Scheinin, Prog. NeuroPsychopharmacol. Biol. Psychiatry, 13 (1989) 635.

2. M. B. Weinger, I. S. Segal, M. Maze, Anesthesiology, 71 (1989) 242.

3. Y. Amemiya, S. S. Hong, B. V. Venkataraman, P. N. Patil, G. Shams, K. Romstedt, D. R. Feller, F-L. Hsu and D. D. Miller, J. Med. Chem., 35 (1992) 750.

4. M. Fatome, H. Sentenac-Roumanou, C. Lion, J. Satgé, M. Fourtinon and G. Rima, Eur. J. Med. Chem., 19 (1984) 119.

5. M. Fatome, H. Sentenac-Roumanou, C. Lion, J. Satgé and G. Rima, Eur. J. Med. Chem., 23 (1988) 257.

6. J. Satgé, G. Rima, M. Fatome, H. Sentenac-Roumanou and C. Lion, Eur. J. Med. Chem., 24 (1989) 48.

7. G. Rima, J. Satgé, C. Lion, H. Sentenac-Roumanou and D. Guyot, Synth. React. Inorg. Met.-Org. Chem., 19 (1989) 787.

8. G. Rima, J. Satgé, M. Fatome, J. D. Laval, H. Sentenac-Roumanou, C. Lion and M. Lazraq, Eur. J. Med. Chem., 26 (1991) 291.

9. G. Rima, J. Satgé, H. Sentenac-Roumanou, M. Fatome, C. Lion and J. D. Laval, Eur. J. Med. Chem., 28 (1993) 761.

10. G. Rima, J. Satgé, H. Sentenac-Roumanou, M. Fatome, J. D. Laval, C. Lion, O. Alazard and P.Chabertier, Appl. Organomet. Chem., 8 (1994) 481.

11. G. Rima, J. Satgé, H. Sentenac-Roumanou, M. Fatome, J. D. Laval, C. Lion and R. Dagiral, Appl. Organomet. Chem., 10 (1996) 113.

12. G. Rima, J. Satgé, H. Sentenac-Roumanou, M. Fatome, J. D. Laval, C. Lion, C. Thiriot, R. Dagiral and C. Martin, Main Group Met. Chem., 20 (1997) 255.

13. G. Rima, J. Satgé, R. Dagiral, C. Lion, Marc Fatôme, V. Roman and Jean-Denis Laval, Metal-Based Drugs, 5 (1998) 139.

14. G. Rima, J. Satgé, R. Dagiral, C. Lion, H. Sentenac-Roumanou, M. Fatôme, V. Roman and J-D. Laval, Metal-Based Drugs, 6 (1999) 49.

15. G. Rima, J. Satgé, R. Dagiral, C. Lion, Marc Fatôme, V. Roman and Jean-Denis Laval, Appl. Organomet. Chem., 13 (1999) 583.

16. J. Satgé, Propriétés et applications biologiques de dérivés organométalliques du silicium, du germanium, et de l'étain, rapport de mise au point A. E. P. A., juin 1981.

17. M.L. Patchen, T.J. Mac Wittie and J.F. Weiss, Int. J. Radiat.Oncol. Biol. Phys., 18 (1950) 286.

18. M.A. Saint-Martino-Descours, M. Cottin and H. Pacheco, Eur. J. Med. Chem. Chim. Ther., 14 (1979) 455.

19. J. Oiry, J. Y. Pue, J. L. Imbach, M. Fatôme, H. Sentenac-Roumanou and C. Lion, J. Med. Chem. 29 (1986) 2217

20. P. Bonet-Maury and F. Patti, J. Radiol. Electrol. Arch. Electr. Med., 31 (1979) 455.

21. D. J. Finney, Probit analysis, Cambridge University Press, Cambridge (1947).

22. M. Lesbre, P. Mazerolles and J. Satgé in: The Organic Compounds of Germanium, John Wiley and Sons, New York, (1973).

23. J. Satgé and M. Baudet, C. R. Acad. Sci. Paris. Ser. C. 263 (1966) 435.

24. J. Satgé, A. Cazes, M. Bouchaut, M. Fatome, H. Sentenac-Roumanou and C. Lion, Eur. J. Med.Chem., 17 (1982) 433.

25. G. Dousse, J. Satgé and M. Rivière-Baudet, Synth. React. Inorg. Met.-Org. Chem., 3 (1973) 11.

26. J. Satgé, M. Lesbre and M. Baudet, C. R. Acad. Sci. Paris. Ser. C. 259 (1964) 4733.

27. M. Unno, Y. Kawai, H. Shioyama and H. Matsumoto, Organometallics, 16 (1997) 4428.

28. J. Corbin, K. F. Miller, N. Pariyadath, S. Wherland, A. E. Bruce and E. I. Stiefel, Inorg. Chim. Acta, 90 (1984) 41.

\section{Received: December 13, 2001 - Accepted: December 17, 2001 - Accepted in publishable format: December 18, 2001}

